\title{
REVISIÓN TAXONÓMICA DEL GÉNERO SUDAMERICANO QUINCHAMALIUM (SCHOEPFIACEAE)
}

\author{
RITA M. LOPEZ LAPHITZ¹ , CECILIA EZCURRA ${ }^{1}$ y ROMINA VIDAL-RUSSELL'1
}

\begin{abstract}
Resumen: El género Quinchamalium comprende hierbas hemiparásitas y es endémico de Sudamérica. Su distribución abarca los Andes desde el norte del Perú hasta el sur de la Patagonia. Los límites morfológicos pocos claros y la escasez de caracteres diagnósticos para la correcta identificación de las especies actualmente aceptadas revelan la necesidad de una revisión de la taxonomía del género. La información morfológica y biogeográfica junto con los análisis morfométricos publicados recientemente señalan la existencia de una sola especie polimórfica: Q. chilense. Como resultado, se presenta un tratamiento taxonómico del género monoespecífico Quinchamalium. Para la única especie del género se provee nueva sinonimia, descripción de características morfológicas vegetativas y reproductivas, y distribución geográfica. En este trabajo, 28 nombres de especies o de taxones infraespecíficos son reducidos a la sinonimia de $Q$. chilense por primera vez. Además, se lectotipifican 13 nombres y uno se neotipifica.
\end{abstract}

Palabras clave: Andes, endemismo sudamericano, especie polimórfica, género monoespecífico, lectotipificaciones, sinonimias nuevas.

\begin{abstract}
Summary: Taxonomic revision of the South American genus Quinchamalium (Schoepfiaceae). The genus Quinchamalium comprises hemiparasitic herbs endemic to South America. Its distribution expands through the Andes from northern Peru to southern Patagonia. The vague morphological limits and the existence of scarce diagnostic characters for the correct identification of the currently accepted species, implies the need for a revision of the taxonomy of the genus. The morphological and biogeographic information along with recently published morphometric analyses indicate the existence of a single polymorphic species: Q. chilense. As a result, a taxonomic treatment of the monospecific genus Quinchamalium is here presented. Valid name, synonyms, description of vegetative and reproductive morphology, and geographical distribution is provided for $Q$. chilense. In this study, 28 names of species or infraspecific taxa are reduced to synonymy of $Q$. chilense for the first time. In addition, 13 names are lectotypified and one is neotypified.
\end{abstract}

Key words: Andes, South American endemism, polymophic species, monospecific genus, lectotypifications, new synonymies.

\section{INTRODUCCIÓN}

En base a los recientes catálogos de Brako \& Zarucchi (1993), de Zuloaga et al. (2009) y de Jørgensen et al. (2014) el género Quinchamalium Molina (Schoepfiaceae) comprende alrededor de 20 especies de hierbas hemiparásitas, distribuidas

\footnotetext{
${ }^{1}$ Instituto de Investigaciones en Biodiversidad y Medioambiente, CONICET UNComahue, Quintral 1250, S. C. de Bariloche, 8400 Río Negro, Argentina, rlaphitz@gmail.com, rlaphitz@comahue-conicet.gob.ar
}

en América del Sur a lo largo de los Andes de Argentina, Bolivia, Chile y Perú (Tabla 1).

El género se ha clasificado tradicionalmente dentro de la familia Santalaceae (Pilger, 1935; Dawson, 1984; Brako \& Zarucchi, 1993; Zuloaga et al., 2009; Kuijt \& Hansen, 2014). Sin embargo, estudios filogenéticos lo ubican en la familia Schoepfiaceae Blume junto a Arjona Cav. y Schoepfia Schreb. (Nickrent et al., 2010).

La asociación de estos tres géneros tempranamente propuesta por Van Tieghem (1896) se ha confirmado con evidencias filogenéticas (Der \& Nickrent, 2008; Vidal-Russell \& Nickrent 2008). 
Aún más, las características florales de estos tres géneros soportan estas relaciones filogenéticas (Nickrent et al., 2010).

Así, los miembros de la familia presentan flores bisexuales en inflorescencias terminales y axilares. Las brácteas y bracteolas florales persisten, en forma separada o fusionadas (calículo: Kuijt \& Hansen, 2014). Rodeando el estilo los tres géneros presentan un disco anular nectarífero (Dawson, 1944). El ovario es ínfero con dos o tres lóculos en la base y unilocular en la parte superior. El fruto en Schoepfia es una drupa mientras que en Quinchamalium y en Arjona es una nuez. Quinchamalium se caracteriza por las brácteas y bractéolas unidas formando una cúpula urceolada, las inflorescencias glabras y los tépalos amarillos, sin pelos detrás de los estambres (Fig. 1). Arjona posee brácteas y bracéolas independientes, inflorescencia pubescente y tépalos blanquecinos o liláceos, con pelos detrás de los estambres (Dawson 1984). Mientras que Quinchamalium y Arjona tienen distribución simpátrica en el sur de Sudamérica, Schoepfia se distribuye en el Neotrópico y Asia.

La taxonomía de todo el género Quinchamalium ha sido poco estudiada. Dada su extensa distribución geográfica, el mismo ha sido básicamente tratado a nivel de revisiones limitadas a enfoques regionales y-o restringidos a límites socio-políticos (por ej., Navas, 1976; Dawson, 1984; Ulibarri, 1994). Mas aún, muchos de los ambientes naturales donde especies nuevas han sido coleccionadas y descriptas en el pasado (Presl, 1849; Philippi, 1857; Miers, 1880) han sido modificados por el hombre por lo que no existen colecciones recientes de esas localidades. Estas razones son suficientes para dificultar los estudios taxonómicos del género. El número de especies que son actualmente reconocidas (Tabla 1) es el resultado de los tratamientos taxonómicos que recopilan información de países individuales. Así, en Argentina en los últimos tiempos se ha reportado una sola especie, Q. chilense (Dawson, 1984, Zuloaga et al., 2009), en Bolivia se han descripto dos especies (Pilger, 1935; Jørgensen et al. 2014), y en Perú seis (Pilger, 1935; Brako \& Zarucchi, 1993) las cuales son consideradas endémicas (Ulloa \& Ulloa, 2006). Finalmente, en Chile se han reportado 14 especies (Philippi, 1857; Dawson, 1944; Zuloaga et al., 2009).

Hasta el momento no existen trabajos sobre la sistemática de este género a una escala que trate la totalidad de su distribución. Por lo tanto, el objetivo principal de este trabajo es resolver la taxonomía de este género sudamericano teniendo en cuenta el reciente estudio de análisis morfométrico multivariado en donde se resolvió la existencia de una sola especie altamente polimórfica en el mismo: Q. chilense Lam. (Lopez Laphitz et al., 2015).

\section{Materiales Y MÉtodos}

Se observaron aproximadamente 750 ejemplares de diferentes herbarios de Argentina, Chile y Estados Unidos: BA, BAB, BCRU, CONC, CORD, LP, MO y SI (siglas según Index Herbariorum; Thiers, 2012). Además, se realizó un viaje de colección a Chile y varios en la Patagonia argentina, en donde se estudió a Quinchamalium en condiciones de campo. El material coleccionado fue depositado en el herbario del Centro Regional Universitario Bariloche (BCRU).

Se consultó la base de datos de fotografías de ejemplares tipo de PlantScience en JSTOR (http:// plants.jstor.org) y la colección de fotografías del Field Museum of Natural History de Chicago (http://emuweb.fieldmuseum.org) de los tipos depositados en el Herbario de Berlín que se destruyeron durante la Segunda Guerra Mundial.

Para las medidas incluidas en la descripción de la especie, se usaron caracteres vegetativos y reproductivos de un subconjunto de 156 de las 750 plantas observadas. El rango de los valores mostrados en la descripción de la especie representa la variabilidad observada en esta muestra de toda la distribución del género (Lopez Laphitz et al., 2015). Para el mapa de distribución se ubicaron todos los especímenes medidos de los cuales la información de localización estaba disponible. En la lista de material representativo se incluyeron estos ejemplares. Algunas fechas de colección no se indican en esta lista.

\section{Resultados y Discusión}

A partir de la información bibliográfica, las observaciones de ejemplares de herbario, las observaciones a nivel poblacional en el campo, los estudios de los patrones de distribución geográfica y 


\section{R. M. Lopez Laphitz et al. - Revisión taxonómica de Quinchamalium}

los análisis estadísticos multivariados de caracteres morfológicos (Lopez Laphitz et al., 2015), así como también de las observaciones de las imágenes de todos los ejemplares tipo, este estudio reconoce la existencia de una sola especie: $Q$. chilense Molina.

Como resultado de la revisión nomenclatural, 28 nombres de especies y-o de taxones infraespecíficos son reducidos a la sinonimia de $Q$. chilense por primera vez, como se señala en la lista sinonímica a continuación con la abreviatura nov. syn. Además, se lectotipifican 13 nombres y uno se neotipifica. En la figura 1 se muestra bajo los nombre $Q$. majus y $Q$. ericoides parte de la diversidad morfológica que llevó a la descripción de un elevado número de especies dentro del género.

\section{Tratamiento taxonómico}

Quinchamalium Molina, Sag. Stor. Nat. Chili: 151-152, 350. 1782. Nom. cons. Especie Tipo: Quinchamalium chilense Molina

Género monoespecífico, endémico de América del Sur, de los Andes desde el norte de Bolivia y Perú hasta el sur de Argentina y Chile.

Obs.: Bentham \& Hooker (1880), Hieronymus (1894) y Dawson (1944) atribuyen el género directamente a Jussieu (1789), el cual menciona a la lámina de Feuillé (1725: 57, lámina 44) como referencia debido a que la descripción de Molina (1782) no es precisa en cuanto al número de semillas en el fruto. Sin embargo, el uso generalizado del nombre Quinchamalium Molina (ej.: Navas, 1976; Zuloaga et al., 2009; Ulibarri, 1994; Missouri Botanical Garden: http:--www.tropicos.org, 28 Jan 2015) apoyan la conservación del nombre ya aprobada (http:--www. bgbm.org-iapt-nomenclature-code-SaintLouis0107AppendixIIIANGEs00303.htm).

Descripción genérico-específica y lista de sinónimos:

Quinchamalium chilense Molina, Sag. Stor. Nat. Chili: 151, 350. 1782. Lam., Tabl. Encycl. ii. 125. t. 142. 1793. Tipo: [Neotypus, aquí designado: lámina 142, Lamarck (1793)]. Obs. 1.

Quinchamalium procumbens Ruiz \& Pav., Fl. Peruv. 2: 1, Ic. 107. 1799 (excl. syn.). Quinchamalium chilense var. procumbens (Ruiz \&
Pav.) Kuntze, Revis. Gen. Pl. 3(3): 284. 1898. Tipo: [Lectotypus, aquí designado: lámina Ic. 107, fig. b, Ruiz y Pavon (1799)]. Obs. 2.

Quinchamalium chilense var. gracile Hook. \& Arn., Bot. Beechey Voy. 44. 1841. Quinchamalium gracile (Hook. \& Arn.) Brongn., Duperrey, Voy. Monde 1829, Atlas Phan. 231. t. 52A. 1826. Tipo: Chile. Valparaíso, Viaje del capitán Beechey, sin colector (Holotypus K 000573545, imagen!). Obs. 3.

Quinchamalium chilense var. robustior Hook. \& Arn., Bot. Beechey. Voy. 44. 1841[1830]. Tipo: Chile. Concepción, Viaje del capitán Beechey, sin colector (Holotypus K 000573543, imagen!), nov. syn.

Quinchamalium majus Brongn., Duperrey Voy. Monde 1829, Atlas. Phan. 229. t. 51A. 1826. Quinchamalium chilense var. majus (Brongn.) Speg., Anales Mus. Nac. Buenos Aires 7: 159. 1902. Tipo: [Lectotypus aquí designado: 1826, lámina 51, Fig. A, Atlas Brongn. Duperrey], nov. syn.

Quinchamalium ericoides Brongn. ex Gay, Hist. Chil. 5: 320. 1846. Tipo: [Lectotypus aquí designado: 1826, lámina 51, Fig. B, Atlas Brongn. Duperrey], nov. syn. Obs. 4.

Quinchamalium elegans C. Presl, Abh. Königl. Böhm. Ges. Wiss., ser. 5, 6: 606. 1851 Epimel. Bot. 246. 1851. Tipo: Chile. Prope Valparaíso, Cuming s.n. [444] [Lectotypus aquí designado: K 000573530, imagen!; isolectotypus K 000573541, imagen!)], nov. syn. Obs. 5

Quinchamalium breviflorum C. Presl, Abh. Königl. Böhm. Ges. Wiss., ser. 5, 6: 606. 1851 Epimel. Bot. 246. 1851. Tipo: Chile. Quillota, Bertero s.n. (Syntypus SGO, no visto); Chile. Cuming s.n. (Syntypus K, no visto), nov. syn. Obs. 6.

Quinchamalium andinum Phil., Bot. Zeitung (Berlin) 15: 747. 1857. Tipo: Chile. Cordillera de Chillán, Germain s.n. [Lectotypus aquí designado: SGO 47724, imagen!], nov. syn. Obs. 7

Quinchamalium pratense Phil., Bot. Zeitung (Berlin) 15: 747. 1857. Tipo: Chile. Tomé, Germain s.n. [Lectotypus aquí designado: SGO 047753, imagen!], nov. syn. Obs. 8.

Quinchamalium excrescens Phil., Bot. Zeitung (Berlin) 15: 748. 1857. Tipo: Chile. Coquimbo, Gay 2023 (Holotypus SGO 047759, imagen!), nov. syn. Obs. 9.

Quinchamalium purpureum Phil., Bot. Zeitung 
(Berlin) 15: 747. 1857. Tipo: Chile. Cordillera de Linares, Germain s.n. [Lectotypus aquí designado: SGO 47795, imagen!], nov. syn. Obs. 10.

Quinchamalium linarioides Phil., Bot. Zeitung (Berlin) 15: 746. 1857. Tipo: Chile. Cordillera de Linares, Germain s.n. [Lectotypus aquí designado: SGO 47726, imagen!], nov. syn. Obs. 11.

Quinchamalium berteroanum Phil., Bot. Zeitung (Berlin) 15: 747. 1857. Tipo: Chile. Santiago, R. A. Philippi s.n. (Holotypus B-10-0248131, imagen!), nov. syn. Obs. 12.

Quinchamalium bracteosum Phil., Bot. Zeitung (Berlin) 15: 747. 1857. Tipo: Chile. Valdivia. $R$. A. Philippi s.n. [Lectotypus aquí designado: B-100248130, imagen!; isolectotypus K 000573536, imagen!] nov. syn. Obs. 13.

Quinchamalium carnosum Phil., Bot. Zeitung (Berlin) 15: 747. 1857. Tipo: Chile. Atacama, Cachinal de la Costa, R. A. Philippi s.n. (Holotypus SGO 47771, imagen!) nov. syn. Obs. 14.

Quinchamalium parviflorum Phil., Bot. Zeitung (Berlin) 15: 747. 1857. Quinchamalium chilense var. parviflorum (Phil.) L. E. Navas. Fl. Cuenca Santiago Chile 2:40. 1976. Tipo: Chile. R. A. Philippi s.n. (Holotypus SGO 47781, imagen!). Obs. 15.

Quinchamalium dombeyi Brongn. ex A. DC., Prodr. 14(2): 626. 1857. Tipo: Probablemente Perú [Lectotypus aquí designado: lámina 52B. Brongn., Duperrey, Voy. Monde Phan. 231. t. (1826)], nov. syn. Obs. 16.

Quinchamalium chilense var. parviflorum A. DC., Prodr. 14(2): 626. 1857. Tipo: Chile. Coquimbo, sin localidad, Gay s.n. (Holotypus G, no visto). nov. syn. Obs. 17.

Quinchamalium thesioides Phil., Bot. Zeitung (Berlin) 15: 747. 1860. Tipo: Chile. Atacama, Hueso Parado, R. A. Philippi s.n. [Lectotypus aquí designado: SGO 47787, imagen!], nov. syn. Obs. 18.

Quinchamalium thesioides var. flaccidum Phil., Fl. Atacama 48. 1860. Tipo: Chile. Atacama, Paposo, R. A. Philippi s.n. (Holotypus SGO 47789, imagen!), nov. syn.

Quinchamalium minutum Phil., Linnaea 33: 233. 1864. Tipo: Chile. Talca, Potrero Grande, Volckmann s.n. (Holotypus SGO 47776, imagen!; isotipo K 000573537, imagen!). nov. syn.

Quinchamalium rugosum Phil., Linnaea 3: 233. 1864. Tipo: Chile. Coquimbo, Choapa, Illapel,
Landbeck s.n. (Holotypus SGO 47777, imagen!), nov. syn.

Quinchamalium litorale Phil., Linnaea 3: 233. 1864. Tipo: Chile. Valparaíso, Aconcagua, Los Molles, XI-1862, Landbeck s.n. (Holotypus SGO 38583, imagen!; isotypus SGO 47794, imagen!), nov. syn.

Quinchamalium patagonicum F. Phil. ex Berg, An. Soc. Cient. Arg.: 143. 1880. Quinchamalium chilense var. patagonicum (Berg) Speg., Anales Mus. Nac. Buenos Aires 7: 159. 1902. Tipo: Argentina. Santa Cruz, Río Santa Cruz, Lago Argentino, Moyano s.n. (Holotypus probablemente LP, no visto). Obs 19.

Quinchamalium tarapacanum Phil., Anales Mus. Nac. Sgo. 75. 1891. Tipo: Chile. Tarapacá, Usmagama, Rahmer s.n. [Lectotypus: aquí designado: SGO 47793, imagen!], nov. syn. Obs. 20

Quinchamalium araucanum F. Meigen., Bot. Jahrb. Syst. 18: 418. 1894. Tipo: Chile. Concepción, R. A. Philippi s.n. (Holotypus B 10-0248132, imagen!), nov. syn.

Quinchamalium stuebelii Hieron., Bot. Jahrb. Syst. 21(3): 306.1896. Tipo: Bolivia. Oruro, 4000 m, 26-X-1876, Stübel 66 [Lectotypus: aquí designado: $\mathrm{B \dagger}$, fotografía FM 14361!]. nov. syn. Obs. 21.

Quinchamalium majus var. spegazzinii Macloskie, Rep. Princeton Univ. Exped. Patagonia, Botany 8: 343. 1905. Tipo: Argentina. Chubut, Río Chubut, Hatcher \& Peterson s.n. (Typus no encontrado). nov. syn. Obs. 22.

Quinchamalium brevistaminatum Pilg., Notizbl. Bot. Gart. Berlin-Dahlem 10: 1030. 1930. Tipo: Perú. Lima, San Agustín, 300-400 m, XII-1910, Weberbauer 5718 (Holotypus B 10-0180508, imagen!; isotypi: F 628698, imagen!, US 1496228, imagen!, S S-R-5414, imagen!). nov. syn.

Quinchamalium elongatum Pilg., Notizbl. Bot. Gart. Berlin-Dahem 10: 1030. 1930. Tipo: Perú. Ocros, Provincia de Cajatambo, Weberbauer 2661 (Holotypus B 10-0180505, fotografía FM 14352!; isotypus $\mathrm{F} 642588$, imagen!). nov. syn.

Quinchamalium hoppii Pilg., Notizbl. Bot. Gart. Berlin-Dahem.10: 1030. 1930. Tipo: PERU. Arequipa, 300-3500 m, VIII-1925. Hopp 56 (Holotypus B 10-0180506, fotografía FM 14354!), nov. syn.

Quinchamalium lomae Pilg., Notizbl. Bot. Gart. Berlin-Dahem. 10: 1030. 1930. Tipo: Perú. Arequipa, Camaná, 280 m., XI-1915, Weberbauer 


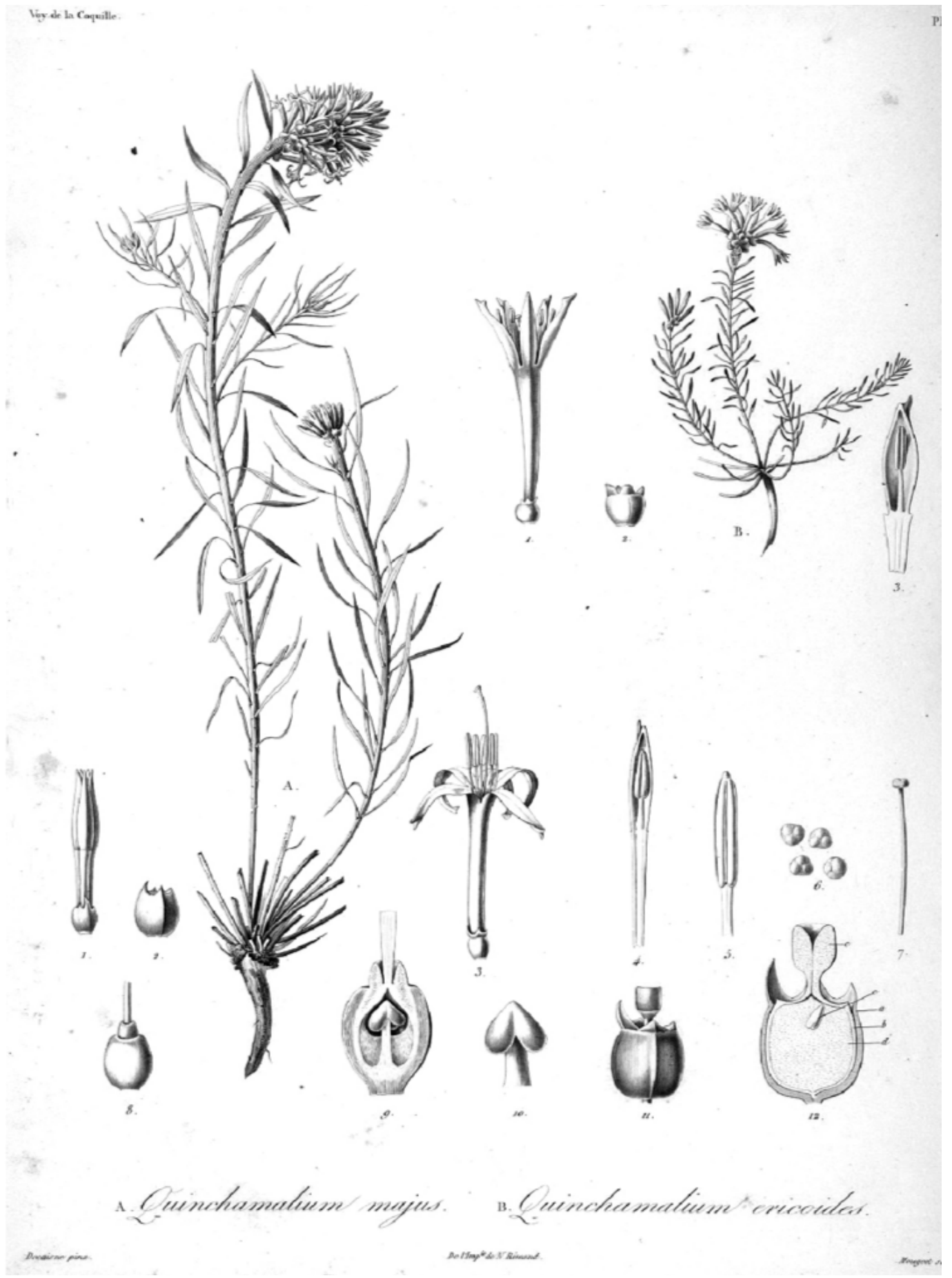

Fig. 1. Quinchamalium chilense (bajo Quinchamalium majus y Q. ericoides). A: Q. majus: Planta; 1, botón floral; 2, calículo; 3, flor; 4, tépalo y estambre (vista ventral); 5 , estambre; 6 , polen; 7 , estilo y estigma; 8 , ovario y nectario (con parte del estilo); 9, corte longitudinal de ovario, nectario y parte inferior del estilo; 10 , óvulos; 11, fruto; 12, corte longitudinal de fruto y semilla. B: Q. ericoides: Planta; 1, flor; 2, calículo; 3, tépalo y estambre (vista ventral) (ilustración de Brongniart,1826) 
7187 (Holotypus B 10-0180507, imagen!; isotypus US 2518193, imagen!), nov. syn.

Quinchamalium raimondii Pilg., Notizbl. Bot. Gart. Berlin-Dahem. 10: 1030. 1930. Tipo: Perú. Raimondi 12552 (Holotypus B 10-0180504, imagen!), nov. syn.

Plantas herbáceas, perennes, de 2,5-40 cm de alto, rizomatosas, hemiparásitas de raíz. Raíz pivotante, notoria, con pequeñas raíces adventicias y haustorios. Tallos erectos o decumbentes, cilíndricos, glabros, pocos ramificados. Hojas sésiles, alternas, aciculares a lineares, glabras, de 7,6-47,4 x 0,4-1,9 mm. Inflorescencias en espigas densas terminales formando cabezuelas, con flores dispuestas sobre el raquis en alveolos rodeados por escamas más o menos desarrolladas. Flores pentámeras, bisexuales, heterostilas, basalmente cubiertas por una cúpula formada por la fusión de una bráctea y dos bractéolas (calículo). Tubo floral (perigonio) alargado, tubuloso, de 0,5-1,8 $\mathrm{mm}$ de longitud, con limbo dividido en 5-tépalos de color amarillo-anaranjado, oblongos, agudos, de 1,7-5,4 x 0,5-1,6 mm con un pequeño espolón en el ápice. Estambres 5, oposititépalos, con filamentos con la porción basal fusionada al tubo floral, de 4-12,2 mm de longitud, y una parte libre de $0,1-1,8 \mathrm{~mm}$; anteras introrsas, biloculares, basifijas, con dehiscencia longitudinal, de 0,2$2,8 \times 0,1-0,7 \mathrm{~mm}$. Ovario ínfero unilocular, con 3 óvulos (se desarrolla uno). Nuez glabra, rodeada por el calículo endurecido de 1,8-3,9 mm de longitud, de forma urceolada, surcado y 3-4 dientes pequeños y uno largo, de 0,3-2 mm de longitud (Fig. 1).

Distribución y hábitat: Especie que se extiende por la región cordillerana de Perú, Bolivia, Chile y Argentina, hasta las estepas arenosas de la zona patagónica andina (Fig. 2). Habita entre los 3800 $\mathrm{m}$ y el nivel del mar, según la latitud.

Fenología: Hierbas con rizoma perenne. Florecen en la temporada estival de Argentina, Bolivia y centro de Chile, mientras que en Perú y el norte de Chile florecen en el período invernal.

Nombre vulgar: "Quinchamalí".

Usos: La especie es utilizada en medicina popular en el sur de Chile (Hoffmann et al., 1992).

Nota. Q. chilense es una especie muy variable en cuanto al porte de la planta, y el tamaño y color de la flor.
Obs. 1: La descripción original de Quinchamalium chilense Molina (1782) menciona las obras de Frézier (1718) y Feuillé (1725) que incluyen ilustraciones de esta especie, sin mencionar material tipo. Lamarck (1793) vuelve a describir la especie citando las dos láminas anteriores (Frézier 1718, Feuillé 1725) y acompaña la descripción con una ilustración de la especie. Se seleccionó como neotipo la imagen de Lamarck (1793), ya que Molina no mencionó material tipo y de estas tres láminas es la única que incluye detalles florales.

Obs. 2: Se seleccionó como tipo de Quinchamalium procumbens a la lámina original de Ruiz \& Pavón (1799). Estos autores citaron dos sintipos, uno de Perú (Huarocherí, Canta y Tarma) y el otro de Chile (Concepción) como material original de Quinchamalium procumbens. Sin embargo, la cartulina de Ruiz y Pavón depositada en el herbario de Madrid (MA 811274, y en la cual no se especifica a cuál de los dos sintipos pertenece) comprende varios ejemplares sin flores desarrolladas. Por lo tanto, el lectotipo elegido aquí es la ilustración que acompaña al protólogo, en la que se observa con claridad las características tanto vegetativas como reproductivas de la especie.

Obs. 3: Se interpreta como holotipo de Quinchamalium chilense var. gracile el ejemplar depositado en K 000573545 debido a que es el que concuerda con la descripción original que menciona "Valparaíso" coleccionada por la expedición del capitán Beechey. Además, está señalado con la letra griega beta la cual está mencionada en el protólogo para esta variedad.

Obs. 4: Gay al describir Quinchamalium ericoides no mencionó ningún ejemplar y basó su descripción en la lámina de Brongniart (1926; Fig. 1), la que se selecciona como lectotipo.

Obs. 5: Se seleccionó como lectotipo de Quinchamalium elegans al ejemplar Cuming 444 (K 000573530), ya que éste se corresponde con el nombre del colector y el lugar de colección del protólogo, aunque en este último no se mencione el número de colección. El ejemplar considerado como isolectotipo (K 000573541 ; ejemplar ubicado en la parte superior a la izquierda de la cartulina) no presenta colector pero si coincide el número de colección.

Obs. 6: Aunque no se han localizado las colecciones de Bertero y Cuming mencionada 


\section{R. M. Lopez Laphitz et al. - Revisión taxonómica de Quinchamalium}

en el protólogo de Quinchamalium Breviflorum. Se sinonimizó este nombre sobre la base de las características que se describen en el protólogo.

Obs. 7: Se seleccionó como lectotipo de Quinchamalium andinum al ejemplar coleccionado por Germain en la Cordillera de Chillán(SGO 47724) dado que presenta mayor número de inflorescencias que el sintipo de Philippi coleccionado en la provincia de Valdivia (B-10-0248133).

Obs. 8: Se seleccionó como lectotipo de Quinchamalium pratense al ejemplar de SGO 047753, coleccionado por Germain en la localidad mencionada en el protólogo en noviembre de 1855 . Existe otro sintipo, HAL 0110972, el cual también coincide con la localidad y la fecha de colección del protólogo (noviembre 1855) pero no es tan completo.

Obs. 9: Se interpreta como holotipo de Quinchamalium excrescens al ejemplar SGO 047759, coleccionado en la localidad típica (Chile, Coquimbo) por Gay, aunque en el protólogo no se asigna un número de colección.

Obs. 10: Se seleccionó como lectotipo de Quinchamalium purpureum al ejemplar SGO 47795 coleccionado en la Cordillera de Linares por Germain, dado que es el único en donde se observan con claridad las características tanto vegetativas como reproductivas de la especie respecto a los otros sintipos: Cordillera de Linares, Germain s.n. (SGO 038588), Cordillera de Linares, R. A. Philippi s.n. (HAL 0111702).

Obs. 11: El ejemplar del herbario de Santiago, SGO 47726, posee dos tallos que parecen provenir de distintas especies. Se eligió como lectotipo de Quinchamalium linarioides la rama de la derecha de esta hoja de herbario, en la cual se observan con claridad las flores respecto de otro sintipo: Cordillera de Linares, R. A. Philippi s.n. (HAL111710).

Obs. 12: Se cita como holotipo de Quinchamalium berteroanum al ejemplar B-10-0248131 ya que fue coleccionado por Philippi en la provincia de Santiago y se corresponde con el protólogo.

Obs. 13: Se seleccionó como lectotipo de Quinchamalium bracteosum a B-10-0248130 debido a que el material fue coleccionado por Philippi en Valdivia, localidad que se menciona en el protólogo. Se identificó como isolectotipo a K 000573536, debido a que el material fue coleccionado en Valdivia, y aunque no se especifica el colector, se observa la escritura perteneciente a Philippi. Otros sintipos: Valdivia, R. A. Philippi s.n. (HBG 510407); Valdivia, R. A. Philippi s.n. (HAL 0111941).

Obs. 14: Se cita como holotipo de Quinchamalium carnosum a SGO47771 debido a que el material fue coleccionado por Philippi en Cachinal de la Costa (Atacama), localidad que se menciona en el protólogo.

Obs. 15: Se cita como holotipo de Quinchamalium parviflorum al ejemplar SGO 47781 debido a que el material fue coleccionado en Hurtado, provincia de Coquimbo, localidad que se menciona en el protólogo.

Obs. 16: A. De Candolle, al describir Quinchamalium dombeyi, no mencionó ningún ejemplar y basó su descripción en la lámina de Brongniart (1826), la que se selecciona aquí como lectotipo.

Obs. 17: Se sinonimizó el nombre de esta variedad (Quinchamalium chilense var. parviflorum) sobre la base de las características que figuran en el protólogo.

Obs. 18: Existen dos ejemplares (SGO 47787 y SGO 47788) que fueron coleccionados en el litoral como menciona el protólogo de $Q$. thesioides. Sin embargo, se seleccionó como lectotipo al ejemplar SGO 47787 (Chile, Hueso Parado) porque está más completo, tanto en cuanto a sus partes vegetativas como reproductivas. Otro sintipo: Paposo, Atacama, Philippi s.n. (SGO 47788).

Obs. 19: La introducción del trabajo de Berg de 1880 especifica que recibió material de esta especie "de mi amigo ... Moyano" coleccionado en las fuentes del Río Santa Cruz, en la costa de un lago desconocido. Aparentemente Moyano hizo viajes al Río Santa Cruz en 1877, 1878 y 1891 , por lo que pudo haberle presentado a Berg material coleccionado en alguno de sus primeros dos viajes. Este material, que fue enviado a $\mathrm{F}$. Philippi desde BA por Berg, hasta ahora no se ha encontrado. La única colección de Moyano de BA es de 1889 (por lo tanto, posterior a la descripción de la especie), y de Chubut (no de Santa Cruz), por lo que no puede considerarse tipo de este nombre.

Se considera sinónimo el nombre $Q$. patagonicum sobre la base de las características que figuran en el protólogo y la sinonimia del Catálogo de las Plantas Vasculares del Cono Sur (Zuloaga et al. 2009). 
Obs. 20: Existen dos ejemplares coleccionados por Rahmer en Usmagama (Tarapacá, Chile) pero con diferentes fechas de colección (13 y 16-III-1885). Dado que ambas se corresponden con el protólogo de Quinchamalium tarapacanum, se seleccionó como lectotipo al ejemplar SGO 47793 debido a que posee mayor cantidad de especímenes y se observan con mayor claridad las características vegetativas y reproductivas que en el sintipo SGO 38582.

Obs. 21: Se seleccionó como lectotipo de Quinchamalium stuebelii la fotografía depositada en $\mathrm{F}$ del ejemplar que estuvo depositado en $\mathrm{B}$ (actualmente destruído), y que coincide con el protólogo en el colector, la fecha y el lugar de colecta. No se localizó ningún otro ejemplar de la colección Stübel 66 de Bolivia, ni del sintipo Stübel 112a de Perú.

Obs. 22: Se considera sinónimo el nombre Q. majus var. spegazzinii sobre la base de las características que se describen en el protólogo.

Material adicional estudiado: ARGENTINA. Prov. Chubut: Sin localidad, Bang 11262 (LP); sin localidad, Hogberg 12349 (SI). Dpto. Languiñeo, Lago Guacho, 11-I-2006, Puntieri 626 (BCRU); Corcovado, Illín 11264 (LP); Represa Futaleufú, Parque Nacional Los Alerces, 7-III-2009, VidalRussell 92 (BCRU). Dpto. Cushamen, sin localidad, 14-I-1947, Soriano 2368 (BAB); Río Tigre, Cholila, Amico 307, 308 (BCRU). Dpto. Futaleufú, Esquel, Alchitt 7372 (BAB). Dpto. Languiñeo, Río Carrenleufú, Soriano 2556 (BAB). Dpto. Río Senguer, Lago Fontana, Alchitt s.n. (BAB). Lago Fontana, Península, 12-XII-1964, M. Clemens s.n. (BA). Prov. Mendoza: Dpto. Malargüe, a $10 \mathrm{~km}$ de la Azufrera, Cabrera et al. 33493 (SI). Sin Dpto. Valle del Yeso, 15-II-1900. G. Gerling (BA). Valle del Lago Blanco, noviembre hasta diciembre, J. Koslowsky s.n. (BA). Prov. Neuquén. Dpto. Aluminé, Aluminé, Crespo \& Giangualani s.n. (BAB); Pulmarí, Cabrera et al. 32924 (SI). Dpto. Huiliches, sin localidad, Correa 5672 (BAB); Paso Pino Hachado, Correa et al. 5625 (BAB); Lago Epulafquen, 4-II-1948, Dawson \& Schwabe 2482 (BAB); Hua-Hum, 4-II-1948, Dawson \& Schwabe 90462 (BAB); subida al cerro de los Angeles, Dawson \& Schwabe 90467 (BAB). Dpto. Lácar, Cerro Chapelco, 14-II-1961, S. Schajowskoi s.n. (BA); Gandullo 162 (BCRU); San Martín de los Andes, Hunziker 6923 (BAB); subida al volcán, Morrone et al. 5711 (BCRU); Cerro Chapelco, Correa 5874 (BAB); San Martín de los Andes, Schajovskoy s.n. (BAB); Parque Nacional Lanín, volcán Lanín, 1-II-2000, Ezcurra et al. 2987 (BCRU); cordón Chapelco, 30-III-2002, Ezcurra 3415 (BCRU). Dpto. Minas, Pichi Neuquén, Boelcke 13624 (SI). Sin Dpto. Cerro Colorado, 03II-1961, Razza s.n. (BA). Quemado del Tromen, Cañadón Grande, 14-XII-1962, Schajowskoi s.n. (BA); Parque Nacional Lanin, Quila-Quina, 23-I1962, Lahitte, Roquero, López s.n. (BA); Las Lagunas, Aduana Vieja, 7-II-1963, Dimitri, Correa Luna, Amorín s.n. (BA); Rahue (Bajada), 23-I1964, Daciuk s.n. (BA); Parque Nacional Lanín, Currhué chico, 21-I-1962, Lahitte, Roquero, López s.n. (BA); Alto río Senguer, 05-XII-1962, Lahitte, Roquero s.n. (BA); Parque Nacional Los Alerces, Lago Futalafquen, 10-XII-1962, Roquero s.n. (BA); Esquel, alrededores, 31-XI-1963, Lahitte, s.n. ( BA). Prov. Río Negro: Dpto. Bariloche, alrededores de S. C. de Bariloche, Kreibhon 326 (SI); Parque Nacional Nahuel Huapi, camino de S. C. de Bariloche a la casa del guardaparque del Río Nirihuau, 10-XII-1996, Ezcurra \& Bianchi s.n. (BCRU); costa del Nahuel Huapi. S. C. de Bariloche, 20-I-1994, Puntieri s.n. (BCRU); valle Río Manso Inferior, 9-XI-1998, Funes \& Arenas s.n. (BAB, BCRU); Parque Nacional Nahuel Huapi, Cuenca del río Manso Superior, I-1969, Diehl Bravo s.n. (BA); costa del Nahuel Huapi, Las Chacras, 10-I2008, Vidal-Russell 60 (BCRU); Ruta Nacional 23, Puesto San Ramón, Vidal-Russell 59 (BCRU); Cajón del Azul, El Bolsón, 27-VIII-2011, Amico 298A,B (BCRU); Cerro López, S. C. de Bariloche, 1-III-1993, Ferreyra 31 (BCRU); S. C. de Bariloche, Co. Ñireco, S. C. de Bariloche, 21-XII-1994, Cadillo \& Posse s.n. (BCRU); Cerro Ñireco, $18 \mathrm{Km}$ al oeste de R.N. 40 hacia Cerro Cachi, Ezcurra \& Ferreyra 3285 (BAB); Parque Nacional Nahuel Huapi, Cerro Ñireco, 23-IV-1949, Montiel s.n. (BA); picada Las Nubes, Réchencq 78a (BCRU); Parque Nacional Nahuel Huapi, Paso de las Nubes, 16-III-1968, Correa Luna s.n. (BA); Cerro Tronador, Parque Nacional Nahuel Huapi, Ezcurra 2281 (BCRU); Cerro Chalhuaco, Nuñez \& Ezcurra 95 (BCRU); Pampa de Huenuleo, S. C. de Bariloche, Temporetti s.n. (BCRU); Parque Nacional Nahuel Huapi, Cántaros a laguna Ortiz Basualdo, Boelcke \& Correa 5327 (BCRU); Parque Nacional Nahuel Huapi, Boelcke \& Correa 5461 (SI). Sin Dpto. 


\section{R. M. Lopez Laphitz et al. - Revisión taxonómica de Quinchamalium}

Pico Jacob, senda A $^{\circ}$ Casa de Piedra, 27-IV-1961, Reynoso, Müller s.n. (BA). Parque Nacional Nahuel Huapi, Cerro Catedral, 10-I-1964, Dimitri \& Correa Luna s.n. (BA); Cañadón de la Mosca, 20-II-1961, M. J. Roquero s.n. (BA); Camino al Crotto, 11-II-1961, Dimitri \& Correa Luna s.n. (BA); Parque Nacional Nahuel Huapi, Lago Gutiérrez, 05-II-1961, Roquero s.n. (BA); Parque Nacional Nahuel Huapi, Camino a Llao Llao Km 4, 15-II-1961, Razza s.n. (BA). Prov. Santa Cruz: Dpto. Lago Argentino, Lago San Martín, Boelcke et al. 16322 (BAB); Lago Argentino, Spegazzini s.n. (BAB). Dpto. Lago Buenos Aires, sin localidad, Boelcke et al. 16131 (BAB). Dpto. Picunches, sin localidad, Carrique 1555 (BAB). Dpto. Río Chico, sin localidad, Boelcke et al. s.n. (BAB). Sin Dpto., Quebrada Río Oro, 03-I-1965, Clemens s.n. (BA). BOLIVIA. Dpto. La Paz: Prov. Copacabana, Copacabana, 7-II-1964, Hunziker 8154 (BAB). Prov. Loyaza, Cerro Quiraya, 22-XII-1987, Lewis 871684A (MO). Prov. Pedro D. Murillo, 2 km sur prov. Palca, 15-III-1987, Solomon 16331 (MO); sin localidad, Nee 33298 (MO); La Paz, Buchtien 511 (MO). Dpto. Oruro: Prov. Litoral, Capi, Bang 761 (MO). CHILE. Región Metropolitana de Santiago (RM): Cerro del Roble, Looser 240 (SI); El Volcán, Cabrera 3461 (LP); El Volcán, 29-XII1935. Cabrera 3461 (BA). Prov. Cordillera, Reserva Nacional Río Clarillo, Romero \& Aldunte 66 (CONC); Puente Manzanito, Aedo 7128 (CONC); Prov. Santiago, San José de Maipo, Mahu 1388-L (LP); norte de Caldera, Santuario Santa Gemita, Muñoz et al. 2859 (MO); Santuario Yerba Loca, estero Yerba Loca, II-2000, Marquez 10 (CONC); Quebrada de Ramón, Tomé 265 (CONC). Región Arica y Parinacota (XV): Prov. Parinacota, entre Pachama y Belén, Belmonte 99229 (CONC); Putre, XI-2009, Aedo 6950 (CONC). Región Tarapacá (I): Prov. Iquique, trayecto entre Chusmiza y Cariquina, IX-1997, Villagrán et al. 9112 (CONC). Región Antofagasta (II): Prov. Antofagasta, Morro Moreno, Schlegel 7809 (CONC); Quebrada La Chimba, Quezada \& Ruiz 79 (CONC); entrada de la Quebrada El Rincón, Quezada \& Ruiz 181 (CONC); dpto. Taltal, Taltal, Wedermann 843 (MO); idem, Johnston 5138 (LP); a $10 \mathrm{~km}$ este de la boca de la quebrada, Worth \& Morrison 15829 (SI). Región Atacama (III): Prov. Atacama, Vallenar, Huasco, Werderman 135 (LP); camino entre Carrizal y
Huasco, Los Toyos, Ackerman 516 (CONC); Prov. Chañaral, Parque Nacional Pan de Azúcar, Dillon 8163 (CONC); Prov. Copiapó, Huasco-Copiapó, $25 \mathrm{~km}$ de Totora, Taylor et al. 10809 (CONC). Región Coquimbo (IV): Prov. Choapa, Pichidangui, Aedo 6835 (CONC); Prov. Elqui, Los Choros, Aedo 6863 (CONC); a $5 \mathrm{~km}$ al sur de Andacollo, Mihoc 416 (CONC); Prov. Limari, camino desde ruta 5 a P.N. Fray Jorge, Tepe et al. 1744 (CONC). Región Valparaíso (V): Prov. Aconcagua, $15 \mathrm{~km}$ de Portillo, Crisci 446 (LP); Prov. Los Andes, Caracoles-Portillo, King 680 (LP); Dpto. Los Andes, camino de los Andes a Portillo, Km 46, Marticorena \& Weldt 575 (LP); Lago Verde, cerca de Valparaíso, Looser 2096 (LP); Termas de Chillán, Ruiz s.n. (LP); Prov. Petorca, Pichicuy, Tepe et al. 1889 (CONC); Prov. Valparaíso, Quintero, Looser 4609 (LP). Región Libertador Bernardo O'Higgins (VI): Prov. Cachapoal, localidad entre Coya y Pangal, Crisci 420 (LP); El Pangal, Zöllner 10143 (CONC); Prov. Cardenal Caro, Tanume, El Peumo, Vera \& Ortiz 63 (CONC); Prov. Colchagua, Termas del Flaco, II1994, Baeza 283 (CONC). Región Maule (VII): Prov. Cauquenes, Reserva Nacional Los Ruiles, XII-1999, Finot \& López 1418 (CONC); Prov. Curicó, cerros antes del camino cortado a El Planchón, I-1995, Marticorena et al. 150 (CONC); Prov. Linares, Res. Nac. Bellotos del Melado, I-2000, Humaña et al. 20093 (CONC); Cerro Maquis, camino Reten Achibueno a Las Animas, III-1999, Ruiz \& López 1078 (CONC); Prov. Talca, Central Los Cipreses, Quebrada El Ciego, IV-2000, Finot \& López 2028 (CONC); Lago del Maule, Boelcke et al. s.n. (BAB); idem, I-1995, Marticorena et al. 232 (CONC); Paso Pehuenche, 30-I-1963, Bacigalupo et al. 10356 (BAB); Reserva Nacional Alto de Vilches, camino a Laguna El Alto, IV-2000, Finot \& López 1859 (CONC). Región Bíobío (VIII): Prov. Arauco, Cordillera Nahuelbuta, Loma del Consorcio, XII1989, Matthei \& Quezada 29 (CONC); Playa Quiala, I-2001, Parra \& Torres 246 (CONC); Trongol Alto, Loma El Consorcio, I-1998, Baeza \& Kottirsch 1352 (CONC); Prov. Bíobio, Antuco, Maldonado 78 (LP); P. N. Laguna Laja, III-2001, Molina 3581 (CONC); volcán Antuco, Las Lagartijas, II-1978, Stuessy \& Baeza 11062 (CONC); Las Huellas, I-1989, Marticorena \& Rodríguez 9989 (CONC); Reserva Nacional 
Ralco, II-2002, Mihoc et al. 4132 (CONC); Prov. Ñuble, Chillán, 7-I-1971, Lourteig 2531 (BAB); camino de Pullay a Quile, XII-2000, Baeza \& López 2269 (CONC); a $2 \mathrm{~km}$ al norte de Colmuyao, XI-2000, Baeza et al. 2060 (CONC); Cerro Ninhue (cumbre), XII-1998, Matthei 608 (CONC). Región La Araucanía (IX): Parque Nacional Malalcahuello, camino a centro de ski Corralco, Morrone 5986 (SI); Prov. Cautín, Trailanquí, Hunckel 866-3 (SI); volcán Lanín, I-1999, Stuessy \& Baeza 15619 (CONC); volcán Villarrica, I-1996, Marticorena et al. 426 (CONC); Prov. Malleco, Cunco, I-1988, Gardner \& Knees 6905 (CONC); Lonquimay, Hollermayer 192 (LP); a $22 \mathrm{~km}$ de Curacautín, camino a Conguillio, $\mathrm{Km} \mathrm{25}$, ruta Interlagos, Morrone 5546 (SI); de Angol a Maitenrehue, Las Bandurrias, I-1988, Baeza \& Kottirsch 1027 (CONC); de Capitán Pastene a El Lingue arriba, I-1998, Baeza \& Kottirsch 1493 (CONC); camino nordeste de Icalma, I-1990, Devore 1129 (CONC). Región Los Lagos (X): Prov. Llanquihue, Parque Nacional Vicente Pérez Rosales, Cerro Riggi, Dawson \& Schwabe 2791 (BAB); desvío de la ruta 65 camino a Lonquimay, Morrone et al 5527 (SI); Prov. Osorno, volcán Antillanca, I-1996, Marticorena et al. 450 (CONC). Región Los Ríos (XIV): Prov. Valdivia, volcán Puyehue, I-1995, Godoy 219 (CONC). Región Aysén del General Carlos Ibáñez del Campo(XI): Prov. Capitán Prat, Cochrane, Lago Chacabuco, I-2007, Teneb 47 (CONC); sector El Manzano, XII-2006, Marticorena et al. 946 (CONC); Puerto Bertrand, I-2007, Jiménez 58 (CONC); confluencia de los ríos Baker y Nadis, I-2007, Teneb 235 (CONC); Prov. Coyhaique, Río Aysén, Burmeister 11269 (LP).

PERU. Dpto. Arequipa: Prov. Arequipa, Solomon 2856 (MO). Dpto. Cajamarca, Celendin, 18-VII-1984, Sagástegui 12169 (MO); Prov. Contumaza, Contumaza, Stuessy et al. 12667 (CONC); Sin localidad, 3-V-1999, Sagástegui 14299 (MO). Dpto. Cuzco: Prov. Calca, VI-1986, Dueñas 111 (MO); Prov. Paruro, Yaurisque, 7-III1987, Nuñez 7419 (MO). Dpto. La Libertad: Prov. Santiago de Chuco, 13-I-1984, Sagástegui 11752 (MO). Dpto. Moquegua: General Sánchez Cerro, 23-III-2009, Montesinos 21669 (MO). Dpto. Tacna: Prov. Tarata, III-1950, Vargas 9244
(MO).

\section{Nombres inválidos o ilegítimos}

Quinchamalium chilense Willd., Sp. P1., ed. 4, 1(2): 1217. 1798. Es un nombre superfluo o ilegítimo porque en la descripción de la especie Willd. se cita al nombre Quinchamalium chilensis de Lamarck.

Quinchamalium peruvianum J. St.-Hil., Expos. Fam. Nat. 1: 175. 1805. Es un nombre inválido por no incluir una diagnosis asociadaen el protólogo.

Quinchamalium tenuis Steud., Nomencl. Bot. ed. 2. ii. 429. 1821-1824. Nomen nudum.

Quinchamalium fructiculosum Steud., Nomencl. Bot. ed. 2. ii. 429. 1821-1824. Nomen nudum.

\section{Nombres excluidos}

Quinchamalium patagonica Spreng., Syst. Veg. (ed. 16) 1: 537. 1824. Es sinónimo de Arjona tuberosa Cav. 1797. Icon. iv. 57. t. 383. (Dawson, 1984; Zuloaga et al. 2008).

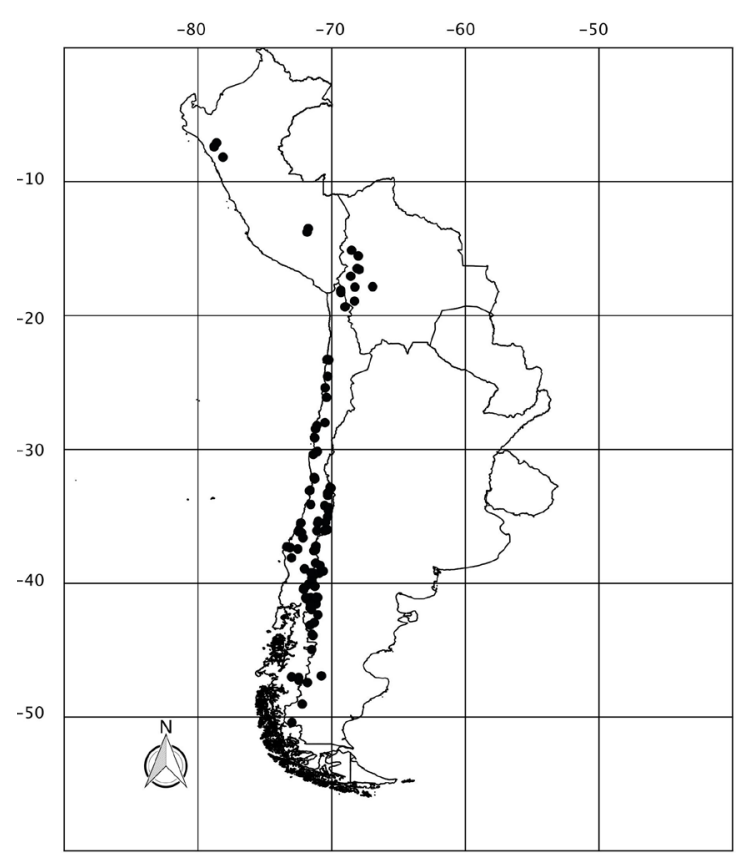

Fig. 2. Mapa del sur y oeste de Sudamérica con la distribución del género Quinchamalium (se ilustran sólo Perú, Bolivia, Chile, Paraguay, Argentina y Uruguay). 
TABLA 1: Especies de Quinchamalium reconocidas hasta 2014 y su distribución según Brako y Zarucchi (1993), Zuloaga et al. (2008) y Jørgensen et al. (2014).

\begin{tabular}{|c|c|c|c|c|}
\hline Nro. & Especie-Variedad & Autor & $\begin{array}{c}\text { Año de } \\
\text { descripción }\end{array}$ & Distribución \\
\hline 1 & Q. andinum & Phil. & 1857 & Chile: Regiones V, VIII, X y XIV \\
\hline 2 & Q. bracteosum & Phi. & 1857 & Chile: Región X y XIV \\
\hline 3 & Q. brevistaminatum & Pilg. & 1930 & Perú: Lima \\
\hline 4 & Q. carnosum & Phil. & 1857 & Chile: Regiones II y III \\
\hline 5 & Q. chilense & Lam. & 1782 & $\begin{array}{l}\text { Argentina: Provincias Chubut, Jujuy, Mendoza, } \\
\text { Neuquén, Río Negro y Santa Cruz } \\
\text { Chile: Regiones I, II, III, IV, V, VI, VII, } \\
\text { VIII, IX, X, XI, XIV, XV y RME } \\
\text { Bolivia: Deptos. Cochabamba, } \\
\text { La Paz, Oruro y Potosí }\end{array}$ \\
\hline 6 & Q. elongatum & Pilg. & 1930 & Perú: Depto. Ancash \\
\hline 7 & Q. excrescens & Phil. & 1857 & Chile: Regiones II, III, IV y V \\
\hline 8 & Q. fructiculosum & Steud. & $1821-1824$ & Chile: Región VIII \\
\hline 9 & Q. hoppii & Pilg. & 1930 & Perú: Depto. Arequipa \\
\hline 10 & Q. linarioides & Phil. & 1857 & Chile: Región VII \\
\hline 11 & Q. linifolium & Meyen & 1878 & Chile: Regiones VII y VIII \\
\hline 12 & Q. litorale & Phil. & 1864 & Chile: Región V \\
\hline 13 & Q. lomae & Pilg. & 1930 & Perú: Depto. Arequipa \\
\hline 14 & Q. parviflorum & Phi. & 1857 & Chile: Regiones IV, VII y RME \\
\hline 15 & Q. pratense & Phil. & 1857 & Chile: Región VIII \\
\hline 16 & Q. procumbens & Ruiz \&Pavón & 1799 & $\begin{array}{l}\text { Perú: Deptos. Ancash, Apurímac, } \\
\text { Arequipa, Cajamarca, Cuzco, Lima, } \\
\text { La Libertad, Moquegua y Tacna }\end{array}$ \\
\hline 17 & Q. purpureum & Phil. & 1857 & Chile: Región VII \\
\hline 18 & Q. tarapacanum & Phil. & 1891 & Chile: Región I y XV \\
\hline 19 & $\begin{array}{l}\text { Q. thesioides } \\
\text { var. thesioides }\end{array}$ & Phil. & 1860 & Chile: Región II \\
\hline 20 & $\begin{array}{l}\text { Q. thesioides } \\
\text { var. flaccidum }\end{array}$ & Phil. & 1860 & Chile: Región II \\
\hline 21 & Q. stuebelii & Hieron. & 1896 & $\begin{array}{l}\text { Perú: Depto. Puno } \\
\text { Bolivia: Depto. Oruro }\end{array}$ \\
\hline
\end{tabular}

\section{Agradecimientos}

Las autoras agradecen a los curadores de los siguientes herbarios: BA, BAB, BCRU, CONC, CORD, LP, MO y SI por su gentileza en facilitar el acceso las colecciones de Quinchamalium y a A. Sérsic, E. Urtubey (SI) y dos revisores anónimos por las valiosas sugerencias realizadas. La primera autora agradece al Consejo Nacional de Investigaciones Científicas y Técnicas (CONICET) de Argentina por el otorgamiento de una beca de doctorado para la realización de este proyecto. Este estudio ha sido posible gracias a las subvenciones otorgadas por las siguientes instituciones científicas argentinas: ANPCyT PICT 2007-1047, CONICET PIP 0282, y UNComahue PIN B149 y B180.

\section{Bibliografía}

BENTHAM, G. \& J. HOOKER. 1880. Genera Plantarum III(1): pg. 220. London.

BRAKO, L. \& J. L. ZARUCCHI. 1993. Santalaceae. En: Catalogue of the Angiosperms and Gimnosperms of Peru 45: 1058-1059. Missouri 
Botanical Garden, St. Louis, Missouri.

BRONGNIART, A. 1826. Atlas. Voyage autour du monde: exécuté par ordre du roi, sur la corvette de Sa Majesté, la Coquille, pendant les années 1822, 1823, 1824, et 1825. Arthus Bertrand, Paris.

DAWSON, G. 1944. Las Santaláceas Argentinas. Rev. Mus. La Plata 6: 5-80.

DAWSON, G. 1984. FAMILIA En: CORREA, M. N. (ed.), Flora Patagónica, Colección Cientifica del Inst. de Tecnología Agrop. 8: 30-42.

DER, J. P. \& D. L. NICKRENT. 2008. A molecular phylogeny of Santalaceae (Santalales). Syst. Bot. 33: 107-116.

FEUILLÉ, L. 1725. Journal des observations physiques, mathématiques et botaniques. Histoire des plantes medecinales qui sont le plus en usage aux royaumes de l'Amerique meridionale, du Perou \& du Chily, composée sur les lieux par ordre du Roy, dans les années 1709, 1710, \& 1711. Pierre Giffart, Paris.

FRÉZIER, A. F. 1718. Relation du voyage de la mer du Sud aux côtes du Chili, du Pérou, et du Brésil, fait pendant les années $1712,1713 \& 1714$. G. Wetstein, Amsterdam.

HIERONYMUS, C. 1894. Santalaceae. En: ENGLER, A. \& K. PRANTL (eds.), Die Natürlichen Pflanzenfamilien, III (1): 306-308. Engelmann, Leipzieg.

HOFFMAN, A., C. FARGA, J. LASTRA \& E. VEGHAZI 1992. Quinchamalí. En: MAGNET, A. (ed.), Plantas medicinales de uso común en Chile, pp. 193-198. Ediciones Fundación Claudio Gay, Santiago, Chile.

JØRGENSEN, P. M., M. H. NEE \& S. G. BECK. 2014. Catálogo de las plantas vasculares de Bolivia. Monogr. Syst. Bot. Missouri Bot. Gard. 127: 1-1744.

JUSSIEU, A. L. de. 1789. Genera plantarum: secundum ordines naturales disposita, juxta methodum in Horto regio parisiensi exaratam. Herrisant, Paris.

KUIJT, J. \& B. HANSEN. 2014. Flowering Plants. Eudicots Santalales, Balanophorales En: KUBITZKI, K. (ed.), The Families and Genera of Vascular Plants, vol. 12.: 143-166. Springer International Publishing, Switzerland.

LAMARCK, J. B. P. A. de M. 1793. Tableau Encyclopedique et Methodique des trois Régnes de la Nature: Botanique, II. Paris.

LOPEZ LAPHITZ, R. M., C. EZCURRA \& R. VIDALRUSSELL. 2015. Morphological variation is associated to climatic patterns along the Andean distribution of Quinchamalium (Schoepfiaceae). Syst. Bot. en prensa.

MIERS, J. 1880. On the Schoepfieae and Cervantesieae, distinct tribes of the Styracaceae. Bot. J. Linn. Soc. 17: 68-87.
MOLINA, J. I. 1782. Saggio sulla Storia Naturale del Chili. Bolonia.

NAVAS, L. E. 1976. Santalaceae. En: Flora de la Cuenca Santiago de Chile 2: DicotyledoneaeArchichlamydeae, pp. 1-40. Chile.

NICKRENT, D. L., V. MALECOT, R. VIDAL-RUSSELL \& J. P. DER. 2010. A revised classification of Santalales. Taxon 59: 538-558.

PHILIPPI, R. A. 1857. Ueber die chilenischen Formen von Quinchamalium. Bot. Zeitung, vol. 15: 745-748. Leipzieg.

PILGER, R. 1935. Santalaceae. En: ENGLER, A. \& K. PRANTL (eds.), Die natürlichen Pflanzenfamilien, vol. 16b: 52-91. Engelman, Leipzieg.

PRESL, C. 1849. Epimeliae Botaniae, pp. 246. Amadei Haase, Praga.

RUIZ, H. \& J. PAVON. 1799. Flora Peruviana, et Chilensis, sive, Descriptiones et icones plantarum Peruvianarum, et Chilensium, secundum systema Linnaeanum digestae, cum characteribus plurium generum evulgatorum reformatisauctoribus. Gabrielis de Sancha, Madrid.

THIERS, B. 2012. Index Herbariorum: A global directory of public herbaria and associated staff. New York Botanical Garden's Virtual Herbarium. http:-sweetgum. nybg. org-ih.. [Acceso: 12 Apbril 2015].

ULIBARRI, E. A. 1994. Santalaceae. En: KIESLING, R. (ed.), Flora de San Juan, República Argentina, vol. I, pp. 289-302. Vázquez Mazzini Editores, Buenos Aires.

ULlOA ULlOA, C. 2006. Santalaceae endémica del Perú. Rev. Per. biol. Número especial 13: 603-604.

VAN TIEGHEM, P. 1896. Sur les phanérogames à ovule sans nucelle, formant le groupedes innucellées ou santalinées. Bull. Soc. Bot. France. 43: 543-577.

VIDAL-RUSSELL, R. \& D. NICKREN. 2008. The first mistletoes: Origins of aerial parasitism in Santalalaes. Mol. Phyl. Evol. 47: 523-537.

ZULOAGA F, MORRONE O \& BELGRANO M. 2008. Santalaceae. En: Catálogo de las plantas vasculares del Cono Sur (Argentina, sur de Brasil, Chile, Paraguay y Uruguay). Monogr. Syst. Bot. Missouri Bot. Gard. 107: 2937-2938. Missouri Botanical Garden Press, Saint Louis, Missouri.

Recibido el 18 de febrero de 2015, aceptado el 13 de abril de 2015 . 